\title{
Method of building transformer acoustic diagnosis database based on multi physical field coupling modeling
}

\author{
Weidong $\mathrm{Wu}^{1}$, Zhengyang $\mathrm{Wu}^{1, *}$, Shouchun $\mathrm{Guo}^{1}$, Yulin $\mathrm{Lu}^{1}$, Li $\mathrm{Ma}^{1}$, and Qinghua $\mathrm{Wang}^{2}$ \\ ${ }^{1}$ Anhui Xiangshuijian Pumped Storage Co. LTD, Wuhu, Anhui, 241083, China \\ ${ }^{2}$ North China Electric Power University, Beijing, 102206, China
}

\begin{abstract}
In this paper, a method of building dynamic acoustic diagnosis database of dry-type transformer is proposed. The method is mainly combined with collecting the basic noise of transformer and simulating the acoustic signals of transformer under different working conditions to improve the fault database of transformer. Due to the incomplete working conditions on site, the simulated working conditions are relatively complete, and the marked acoustic signal file is generated, and then form the composition of the database. This paper focuses on the finite element simulation analysis of transformer vibration and noise radiation based on COMSOL, and the idea of establishing transformer biological diagnosis database. Improve the efficiency of transformer fault design and research, and provide reliable and convenient data services for researchers.
\end{abstract}

\section{Introduction}

In the power system, as one of the important power equipment, transformer has a large number, long operation time and many kinds of specifications, so the failure rate is high. According to the statistics of state grid equipment accidents ${ }^{[1]}$, in 2005 , the accident rate of $110 \mathrm{kV}$ and above transformers was as high as 18 , which led to the equipment outage maintenance and economic loss of more than 100 million yuan. Some experienced staff can experience when inspecting the power transformer: the noise is stable in normal operation; when the transformer fails or external disturbance occurs, the noise will change to a certain extent, such as "bang", "Pa Pa Pa", "buzz" and so on ${ }^{[2]}$. This auscultation method is difficult to be widely used because of its legal factor. With the continuous development of transformer acoustic diagnosis technology, noise analysis method ${ }^{[3-6]}$, acoustic emission analysis technology and ultrasonic analysis method ${ }^{[7]}$ are widely used.

Transformer fault database is an important platform for the research of audible transformer fault diagnosis. However, the lack of integrated database tools in this field limits the efficiency of transformer fault design and research. In this paper, a method of building acoustic database of transformer is designed to simulate the sound data of transformer in different operation states in multi physical field simulation environment, which provides data

*Corresponding author: 1269567697@qq.com 
acquisition method support for audible transformer fault diagnosis platform.

\section{Simulation calculation principle}

\subsection{Magnetic field analysis of transformer with multiple physical fields}

Maxwell's equations are the basis of electromagnetic field theory. Ampere's loop law, Faraday's electromagnetic induction law, Gauss's electric flux law and Gauss's magnetic flux law constitute Maxwell's equations ${ }^{[8]}$. In the electromagnetic field, the differential form of Maxwell equations is often used in the finite element method:

$$
\left\{\begin{array}{l}
\nabla \times H=J+\frac{\partial D}{\partial t} \\
\nabla \times E=-\frac{\partial B}{\partial t} \\
\nabla \cdot D=\rho \\
\nabla \cdot B=0
\end{array}\right.
$$

Where, $H$ is magnetic field strength; $B$ is magnetic induction strength; $E$ is Electric field strength; $J$ is current density. $D$ is the field quantity equal to $\varepsilon E$, and $\varepsilon$ is dielectric constant.

\subsection{Analysis of solid mechanical field in transformer multi physical field}

The entity in structural mechanics module is selected for structural field analysis, and the stress-strain (smsld) is set as $(\mathrm{u}, \mathrm{v}, \mathrm{w})$. The equation is solved in the solution domain with transient damping:

$$
m \frac{d^{2} u}{d t^{2}}+\xi \frac{d u}{d t}+k u=f
$$

Where, $\mathrm{u}$ is displacement; $\xi$ is damping parameter; $k$ is stiffness matrix.

\subsection{Analysis of multi physical field pressure acoustic field of transformer}

In continuous media, the working state and pressure change of any point in the sound field can be expressed as sound pressure. The actual sound field in engineering is usually complex, but it can be approximately decomposed into a combination of several typical sound source models. For the calculation of the sound field of the transformer in this paper, the iron core can be regarded as a combination of monopole and dipole sound source models. In this way, the governing equation of the solution domain in the sound field analysis is obtained as follows:

$$
\frac{1}{\rho c^{2}} \frac{\partial^{2} p_{t}}{\partial t^{2}}+\nabla \cdot\left(-\frac{1}{\rho}\left(\nabla p_{t}-q_{d}\right)\right)=Q_{m}
$$

Among them, $\rho$ is fluid density; $c$ is sound velocity; $p_{t}$ is absolute sound pressure; $q_{d}$ is dipole source; $Q_{m}$ is monopole source. Through the coupling calculation of solid mechanics field and sound field, the distribution and magnitude of sound pressure level of transformer 
noise in air cavity can be analyzed and calculated.

\section{Simulation modelling and simulation results}

Simulation needs geometric modelling, setting material and physical field, meshing, solving and post-processing . The specific process is shown in Figure 1:



Fig. 1. Simulation modelling process.

This study takes a $35 \mathrm{kV}$ dry-type power transformer with capacity of $80000 \mathrm{kVA}$ as the calculation object to carry on the simulation analysis. The main parameters of the power transformer are as follows: rated voltage at high voltage side is $35 \mathrm{kV}$, winding inner diameter is $440 \mathrm{~mm}$, winding outer diameter is $555 \mathrm{~mm}$, winding height is $780 \mathrm{~mm}$; the inner diameter of winding on low voltage side is $283 \mathrm{~mm}$, winding outer diameter is $370 \mathrm{~mm}$, winding height is $1360 \mathrm{~mm}$.

After the geometric model is built, the material definition is made for each part of the transformer. For the transformer model, the core, winding and air materials are respectively set as air, Cooper and soft iron. In order to conduct engineering analysis of transformer model, some attributes need to be defined, as shown in Figure 1:

Table 1. Main parameters of material properties.

\begin{tabular}{|ccccc|}
\hline Structural & Material & Property & Value & Unit \\
\hline \multirow{4}{*}{ Winding } & & Relative permeability & 1 & \\
& \multirow{3}{*}{ Copper } & Relative permittivity & 1 & \\
& & Density & 8940 & $\mathrm{~kg} / \mathrm{m}^{3}$ \\
& & Poisson's ratio & 0.34 & \\
& & Young's modulus & $126 \mathrm{e} 9$ & $\mathrm{~Pa}$ \\
\hline \multirow{3}{*}{ Iron core } & \multirow{3}{*}{ Soft iron } & Conductivity & 5 & $\mathrm{~s} / \mathrm{m}$ \\
& & Relative permittivity & 1 & \\
& & Density & 7870 & $\mathrm{~kg} / \mathrm{m}^{3}$ \\
& & Poisson's ratio & 0.28 & \\
& & Young's modulus & $2 \mathrm{e} 11$ & $\mathrm{~Pa}$ \\
\hline
\end{tabular}

After meshing, the model consists of 22250 domain elements, 5170 boundary elements 
and 1070 edge elements. Considering the calculation time and accuracy, the physical field control grid is used. The direct method is used to solve the linear solver. Because this simulation involves multiple physical fields coupling, the convergence condition is harsh, so the limit of iteration times is increased. The coil is set as current excitation, and sinusoidal current with amplitude of $132 \mathrm{~A}$ and $733 \mathrm{~A}$ and frequency of $50 \mathrm{~Hz}$ is used as excitation. The data calculation results are shown in the figure below:

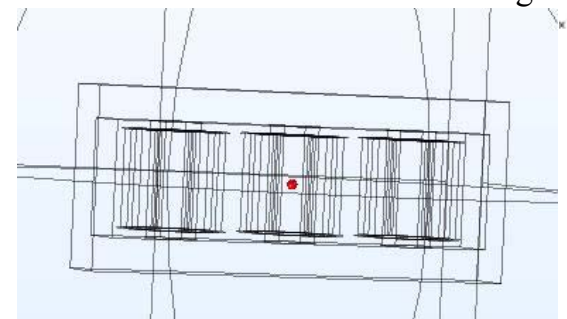

a. Core displacement sampling point

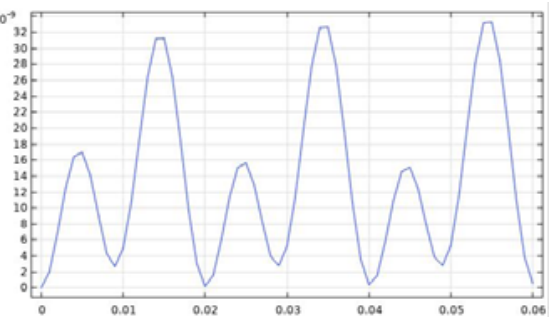

b. Displacement of sampling point

Fig. 2. Three dimensional section point 1 and the acceleration at that point.
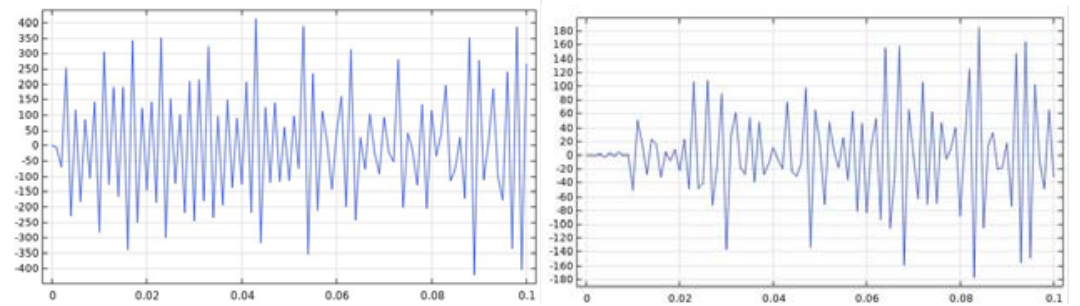

a. Sound pressure at a point in front of transformer b. Sound pressure at a point above the transformer

Fig. 3. Sound pressure at different sampling points.

Under rated conditions, as shown in Figure 9, the core displacement collected at the core is basically $100 \mathrm{~Hz}$, and the main frequency of displacement change during normal operation of the transformer obtained under the action of magnetostriction is $100 \mathrm{~Hz}$, as shown in Figure 10 , because the sound wave is a standing wave, the arrival time of the sound wave is not completely consistent according to the position of the point, and the waveform comparison law of the two figures remains basically consistent. There is no big difference between the two. The finite element simulation of the transformer constructs the sound field distribution map of the air region around the transformer. According to the distribution map of the sound pressure level at different times, it can be seen that the sound field distribution presents a certain symmetrical distribution characteristics. At the same time, the vibration and noise distribution data of the transformer at each time are accurately obtained. In the future, it can be applied to neural network training and fault identification on the basis of data set formed by marked acoustic signal files under different working conditions of transformer.

\section{Conclusion}

This paper focuses on the analysis of using COMSOL multiphysics finite element simulation software to establish the multi physical field simulation model of the transformer, focusing on the vibration displacement deformation of the transformer structure in the magnetic field, and the noise distribution caused by the structure vibration. The noise of transformer is mainly caused by the vibration of winding and core in electromagnetic field. Through the analysis of the principle of transformer core magnetostriction and the characteristic source of transformer vibration noise, the most important source is the magnetostrictive effect of core silicon steel sheet. The fundamental frequency of vibration and noise caused by 
magnetostriction is $100 \mathrm{~Hz}$, and the fundamental frequency of vibration acceleration of iron core is $100 \mathrm{~Hz}$; In this paper, the magnetic field, solid mechanics field and sound field are analytical theoretically and applied to the coupling calculation of multi physical fields; In this paper, the vibration characteristics and noise mechanism of the transformer are combined with the simulation research, and the relationship between the vibration and noise of the transformer is analyzed. By using COMSOL multiphysics to simulate the three-dimensional transient of three-phase dry-type transformer, the effective coupling analysis of magnetic field, solid mechanics field and sound field is realized; The finite element simulation of the transformer constructs the sound field distribution map of the air region around the transformer. According to the distribution map of the sound pressure level at different times, it can be seen that the sound field distribution presents a certain symmetrical distribution characteristics. At the same time, the vibration and noise distribution data of the transformer at each time are accurately obtained. In the simulation of this paper, only the transient numerical calculation is realized. In the frequency domain analysis, the spectrum analysis of vibration and noise can be realized, and the vibration displacement and noise sound pressure level under different frequencies can be studied.

\section{Acknowledgement}

This work was supported by the project of development and application of acoustic monitoring and diagnosis device for power transformer in Xiangshuijian hydropower station under Grants 52572920000a.

\section{References}

1. Wang Mengyun. Statistical analysis of $110 \mathrm{kV}$ and above transformer accidents in 2004 [J]. Power equipment, 2005 (11): 35-41.

2. Liu Song. Measurement of magnetic properties and analysis of electromagnetic vibration and noise of transformer core materials [D]. Tianjin University of technology, 2019.

3. Huang Zhiming, Wang Qiao, Zhang Yifan, Jing Yi, fuminli, Zhuoren, Luo Yan, Feng Feng, Ning Wenjun. Simulation analysis of stress and vibration characteristics of $500 \mathrm{kV}$ connecting transformer under harmonic condition [j]. High voltage electrical apparatus, 2019,55 (11): 86-92.

4. Yi Feng, Liang Jian, Xu Wei, Gao Zhixin, Ma Guoqing. Analysis on abnormal partial discharge of $500 \mathrm{kV}$ Transformer [J]. Shandong electric power technology, 2020,47 (08): 10-13.

5. Wang Zezhong, Li Bing, Liu Haibo, Li Haiming, Meng Hui. Experimental study on vibration and noise of $500 \mathrm{kV}$ single phase transformer under DC bias [J/OL]. Acta electrotechnics Sinica,2020: 1-11.

6. Li Dajian, Yu Changting, Chen Liangyuan, Yan Haijun. Statistical analysis of vibration characteristics of power transformers with different voltage levels [J]. High voltage apparatus, 2019,55 (11): 118-125.

7. Wei J.Fan L.Zhiyuan W.et al. Transformer Partial Discharge Detection Using ElectricalUltrasonic Technology[C]. 2012.

8. Chen Yongshuai. Research on intelligent noise control system of power transformer [D]. Shandong University, 2020. 